\title{
Perfil sociodemográfico e avaliação neurofuncional de mulheres no pós-alta de
}

\section{hanseníase}

\author{
Sociodemographic profile and neurofunctional evaluation of women in the pos-release of leprosy \\ Perfil sociodemográfico y evaluación neurofuncional de mujeres em post liberación de lepra
}

Recebido: 09/11/2021 | Revisado: 18/11/2021 | Aceito: 26/11/2021 | Publicado: 07/12/2021

\author{
Ana Beatriz de Almeida Lima \\ ORCID: https://orcid.org/0000-0001-6588-1070 \\ Universidade de São Paulo, Brasil \\ E-mail: ana.beatriz_al@usp.br \\ Clodis Maria Tavares \\ ORCID: https://orcid.org/0000-0001-6804-3064 \\ Universidade Federal de Alagoas, Brasil \\ E-mail: clodistavares@yahoo.com.br \\ Tamyssa Simões dos Santos \\ ORCID: https://orcid.org/0000-0002-7911-0389 \\ Centro Universitário Mario Pontes Jucá, Brasil \\ E-mail: simoestamyssa@gmail.com \\ Fernanda Silva Goes \\ ORCID: https://orcid.org/0000-0003-1547-7410 \\ Secretaria Municipal de Saúde de Porto de Pedras, Brasil \\ E-mail: fernanda.goes_@outlook.com \\ Nayara Figueiredo Vieira \\ ORCID: https://orcid.org/0000-0001-6218-1394 \\ Universidade Federal de Goiás, Brasil \\ E-mail: nayaravieira@ufg.br \\ Alessandra de Almeida Silva Figueredo \\ ORCID: https://orcid.org/0000-0003-3758-8613 \\ Secretaria Municipal de Saúde de Maceió, Brasil \\ E-mail: alehtalmeida@gmail.com
}

\begin{abstract}
Resumo
Objetivo: Avaliar a situação neurofuncional simplificada em mulheres no pós-alta de hanseníase em um município de Alagoas, tendo em vista que essas alterações podem afetar o cotidiano das mulheres e suas relações familiares e sociais. Método: Estudo transversal e descritivo, no qual foi realizada avaliação neurofuncional em mulheres no pósalta de hanseníase e aspectos sócio demográficos. Foram calculadas frequências relativas e absolutas utilizando-se o Excel. Resultados: Aos aspectos sócio demográficos, maioria era dona de casa, parda, ensino fundamental I e autorreferiu receber até 1 salário mínimo e meio. Na avaliação neurofuncional, foi encontrada maior ocorrência de alterações sensitivas em comparação as motoras e também ausência de deformidades físicas. Conclusão: Constatou se fragilidade do serviço em acompanhar as mulheres no pós- alta por cura de hanseníase, tendo em vista as repercussões psicológicas, físicas e sociais que a doença pode causar em maior intensidade no sexo feminino. No período pós-alta, prevenção e manejo de incapacidades residuais ainda se constituem como desafio, pois as pacientes já concluíram o tratamento.
\end{abstract}

Palavras-chave: Hanseníase; Saúde da mulher; Doenças negligenciadas; Enfermagem; Saúde da pessoa com incapacidade.

\begin{abstract}
Objective: To evaluate the simplified neurofunctional situation in post-discharge women from leprosy in a city in Alagoas, considering that these changes can affect the daily lives of women and their family and social relationships. Method: Cross-sectional and descriptive study, in which a neurofunctional assessment was carried out in postdischarge women from leprosy and socio-demographic aspects. Relative and absolute frequencies were calculated using Excel. Results: Regarding socio-demographic aspects, most were housewives, mixed race, elementary school I and self-reported receiving up to 1 and a half minimum wage. In the neurofunctional evaluation, a higher occurrence of sensory alterations was found in comparison with the motor ones, as well as the absence of physical deformities. Conclusion: It was found that the service was weak in following up post-discharge women due to a cure for leprosy, in view of the psychological, physical and social repercussions that the disease can cause to a greater extent in females. In the post-discharge period, prevention and management of residual disabilities are still a challenge, as the patients have already completed the treatment.
\end{abstract}

Keywords: Leprosy; Women's health; Neglected diseases; Nursing; Health of the disabled. 


\section{Resumen}

Objetivo: Evaluar la situación neurofuncional simplificada en mujeres post alta por lepra en una ciudad de Alagoas, considerando que estos cambios pueden afectar la vida cotidiana de las mujeres y sus relaciones familiares y sociales. Método: estudio descriptivo transversal, en el que se realizó una valoración neurofuncional en mujeres post egreso por lepra y aspectos sociodemográficos. Las frecuencias relativas y absolutas se calcularon utilizando Excel. Resultados: En cuanto a aspectos sociodemográficos, la mayoría fueron amas de casa, mestizos, primaria I y autoinformaron percibir hasta 1 salario mínimo y medio. En la evaluación neurofuncional se encontró una mayor ocurrencia de alteraciones sensoriales en comparación con las motoras, así como la ausencia de deformidades físicas. Conclusión: Se encontró que el servicio fue deficiente en el seguimiento de las mujeres post alta por cura de la lepra, ante las repercusiones psicológicas, físicas y sociales que la enfermedad puede causar en mayor medida en las mujeres. En el período posterior al alta, la prevención y el manejo de las discapacidades residuales siguen siendo un desafío, ya que los pacientes ya completaron el tratamiento.

Palabras clave: Lepra; Salud de la mujer; Enfermedades desatendidas; Enfermería; Salud de la persona com discapacidad.

\section{Introdução}

A hanseníase é uma doença infectocontagiosa, cujo agente etiológico é o Mycobacterium leprae conhecido como Bacilo de Hansen. Essa bactéria possui tropismo por pele e nervos periféricos, pode provocar deformidades e incapacidades físicas nas pessoas acometidas. A doença ainda representa um problema de saúde pública mundial, devido a magnitude, poder incapacitante e concentrada em grupos populacionais vulneráveis (Leal et al., 2017). Está intimamente ligada às condições socioeconômicas dos indivíduos, além de carregar um estigma social, que dificulta a obtenção do diagnóstico precoce, ocasionando assim, disseminação do bacilo nas famílias e comunidades, além disso, faz parte da lista de doenças negligenciadas (Brasil, 2016a; Pinheiro, et al., 2019).

Segundo a Organização Mundial da Saúde, anualmente são registrados cerca de 200 mil casos novos de hanseníase no mundo. No entanto, no ano de 2020 observou-se uma redução de 38,1\% na detecção de casos novos em relação a 2019, em função da pandemia do novo coronavírus. Em termos absolutos, Índia e Brasil, são os países com maior número de casos novos, concentrando cerca de $70 \%$ do total (WHO, 2021). No Brasil, o desafio é quebrar a cadeia de transmissão da doença, no entanto, é preciso qualificar os serviços de saúde.

Em 2019, a taxa de detecção de hanseníase na região nordeste foi de 19,97 casos/100 mil habitantes e em menores de 15 anos foi de 5,37 casos/100 mil habitantes, ambos indicadores apontam muito alta endemicidade (Brasil, 2021). A Secretaria de Estado da Saúde de Alagoas (SESAU) registrou 334 novos casos de hanseníase, número maior ao ano anterior, 2017, que somaram 307 casos (Alagoas, 2019). Rio Largo, município de Alagoas, desde 2003, tem apresentado alta endemicidade em hanseníase, com maior taxa de detecção em 2007, correspondente a 22,92/100 mil habitantes. Quanto aos dados de pessoas que tiveram hanseníase do sexo feminino, o município de Rio Largo apresentou uma média de 40\% do total de pessoas atingidas, entre o período de 2000 e 2015 (Alagoas, 2015).

Por ser uma doença de prevalência em países subdesenvolvidos e em desenvolvimento, como é o caso do Brasil, a hanseníase também se destaca entre as Doença Tropical Negligenciada (DTN), que acomete principalmente populações pobres e grupos vulneráveis. Além disso, dentro dessa conjuntura se destaca também as questões de gênero, nos quais as mulheres estão inseridas (Ávila e Silva \& Barsaglini, 2018).

Para Gonçalves et al. (2018), a mulher afetada pela hanseníase apresenta dupla vulnerabilidade, relacionadas as desigualdades de gênero e aos padrões de beleza impostos pela sociedade, isso interfere no cotidiano social das mulheres acometidas pela doença. Além disso, as características do gênero feminino, como serem cuidadoras da família, desempenhando papéis de "dona de casa" e "mãe", essas mulheres negligenciam a própria saúde e priorizam as tarefas domésticas (Lima et al., 2021). A doença é carregada de estigma, e quando mulheres são afetadas pela hanseníase, suas atividades laborais e domésticas podem ser impactadas, uma vez que a doença pode causar dores físicas e incapacidades. 
Segundo Gonçalves et al. (2018), a diminuição da produtividade pode acarretar a perda de emprego formal e assim causar danos financeiras e emocionais a essas mulheres.

$\mathrm{Na}$ conjuntura da mulher brasileira, a hanseníase causa alterações físicas devido as manifestações clínicas, pode modificar a cor da pele em função da poliquimioterapia, traz impactos psicológicos pelo diagnóstico, e também, por ser mulher e ter as funções afetadas, tudo fica exacerbado (Gonçalves, et al., 2021).

Além disso, as alterações hormonais da puberdade, gestação, puerpério e menopausa, bem como o estado fisiológico de imunodepressão, provocam reflexos nas mulheres acometidas pela hanseníase. As mulheres infectadas pelo Mycobacterium leprae, provavelmente apresentam os primeiros sinais e sintomas no período da gestação ou puerpério. No entanto, quando a doença é presente anterior ao período gravídico-puerperal, existe uma piora do caso, devido à redução da imunidade que pode aumentar índices de recidiva e reações (Tavares, 2014).

Assim, o presente estudo tem como objetivo avaliar a situação neurofuncional simplificada em mulheres no pós-alta de hanseníase em um município de Alagoas, tendo em vista que essas alterações podem afetar o cotidiano das mulheres e suas relações familiares e sociais.

\section{Metodologia}

\section{Tipo de estudo}

Trata-se de um estudo transversal, descritivo e com abordagem quantitativa.

O estudo transversal de abordagem quantitativa possibilita estudar uma determinada população em um dado momento, além de trabalhar com amostra, prevalência, testes diagnósticos, avalia a relação entre as variáveis e associação de risco (Capp \& Nienov, 2020). Além disso, os dados podem ser coletados através de fontes diretas também chamadas de primárias ou a partir de fontes secundárias (Freire \& Pattussi, 2018). Descreve assim, a distribuição da doença, incluindo consideração sobre população ou subgrupo que desenvolvem ou não a doença, em qual localidade geográfica é mais comum e como a frequência da ocorrência varia com o tempo (Bonita, et al., 2010).

\section{Cenário}

A coleta de dados foi realizada em uma Unidades Básica de Saúde (UBS) do município de Rio Largo em Alagoas, no período de abril a julho de 2019. O município de Rio Largo tem uma população estimada em 2021 de 75.662 pessoas. Segundo informações do Instituto Brasileiro de Geografia e Estatística (IBGE), a taxa de mortalidade infantil média na cidade é de 12,78 para 1.000 nascidos vivos e o Índice de Desenvolvimento Humano (IDH) de 2010 foi de 0,643 (IBGE, 2021).

\section{Fonte de dados}

Foi construído um instrumento para avaliar a função neurofuncional das mulheres, no qual havia questões sócio demográficas, aspectos clínicos- epidemiológicos e avaliação neurológica simplificada. A aplicação do instrumento de avaliação da situação neurofuncional simplificada foi realizada durante as consultas de enfermagem.

\section{Participantes do estudo}

Participaram do estudo mulheres diagnosticadas com hanseníase de 2007 a 2017, maiores de 15 anos e residentes no município de Rio Largo. Foram elegíveis para o estudo 46 mulheres, nos quais foram excluídas 01 por erro diagnostico, 02 óbitos e 03 transferências para outros municípios. Das 40 mulheres restantes, houveram 03 recusas de participação do estudo e 16 não foram localizadas. Dessa maneira, a população de estudo foi composta por 21 mulheres no pós-alta de hanseníase. 


\section{Análise dos dados}

Os dados foram inseridos em planilha do Microsoft Office Excel® 2016, e posteriormente, foi realizada análise descritiva das variáveis por meio do programa Statistical Package for the Social Sciences (SPSS), versão 21.0. Para análise dos dados, foram construídas tabelas com frequência absoluta e relativa.

\section{Aspectos éticos}

O estudo foi aprovado pelo Comitê de Ética em Pesquisa da Universidade Federal de Alagoas, sob parecer CAAE $\mathrm{n}^{\circ}$ 02327118.4.0000.5013. Todos os participantes assinaram o Termo de Consentimento Livre e Esclarecido (TCLE) foram informados sobre a garantia de desistência da pesquisa a qualquer momento, conforme preconiza a resolução 510 do Conselho Nacional da Saúde do Ministério da Saúde (2016), bem como da livre e espontânea participação.

\section{Resultados e Discussão}

Participaram do estudo 21 mulheres, dos quais $42,86 \%$ (n=9) estavam na faixa etária acima dos 55 anos; 55,17\% $(\mathrm{n}=12)$ relataram ser dona de casa; 52,38\% $(\mathrm{n}=11)$ autorreferiram cor parda. E 28,57\% $(\mathrm{n}=6)$ responderam ser analfabetas, sendo que cerca de $90 \%$ referiram renda de um salário mínimo e meio (Tabela 1).

Tabela 1: Perfil sociodemográfico das mulheres pós-alta de hanseníase participantes do estudo. Rio Largo-AL, Brasil, $2019(\mathrm{n}=21)$.

\begin{tabular}{llrr}
\hline Variáveis & Categorias & N & \% \\
\hline \multirow{4}{*}{ Idade } & Entre 18 e 25 anos & 2 & 9,52 \\
& Entre 26 e 35 anos & 2 & 9,52 \\
& Entre 36 e 45 anos & 4 & 19,05 \\
& Entre 46 e 55 anos & 4 & 19,05 \\
& Acima de 55 anos & 9 & 42,86 \\
\hline \multirow{4}{*}{ Ocupação } & Aposentada & 3 & 14,29 \\
& Dona de casa & 12 & 57,14 \\
& Autônoma & 3 & 14,29 \\
& Diarista & 2 & 9,52 \\
& Recepcionista & 1 & 4,76 \\
\hline \multirow{4}{*}{ Cor } & Branca & 4 & 19,05 \\
& Preta & 4 & 19,05 \\
& Amarela & 2 & 9,52 \\
& Parda & 11 & 52,38 \\
\hline \multirow{3}{*}{ Escolaridade } & Analfabeta & 6 & 28,57 \\
& Ensino Fundamental I $\left(1^{\circ}\right.$ ao $5^{\circ}$ ano $)$ & 7 & 33,33 \\
& Ensino Fundamental II $\left(6^{\circ}\right.$ ao $9^{\circ}$ ano $)$ & 3 & 14,29 \\
& Ensino Médio & 5 & 23,81 \\
\hline \multirow{2}{*}{ Renda } & Até 1 salário mínimo e meio & 19 & 90,48 \\
& Acima de 1 salário mínimo e meio & 2 & 9,52 \\
\hline
\end{tabular}

Fonte: Autores (2019).

Evidências científicas têm apontado a associação da hanseníase com condições socioeconômicas desfavoráveis, como por exemplo, a relação do aumento no nível de escolaridade dos casos com a redução da chance de diagnostico com alguma 
incapacidade instalada (Rodrigues et al., 2017; Monteiro et al., 2017; Lages et al., 2018). Os resultados desse estudo também indicam que no sexo feminino a doença acomete a população vulnerável, destacando-se nas participantes o analfabetismo e a baixa renda. Cerca de 38,2\% dos casos novos de hanseníase diagnosticados no mundo são em mulheres (WHO, 2021), no entanto, apesar disso é preciso pensar em estratégias apropriadas e específicas para a população feminina. Pois estas representam risco duplo de vulnerabilidade, que se relaciona ao gênero e as condições socioeconômicas. Além disso, mulheres em idade fértil que são acometidas pela doença, tem maior risco de desenvolver reações hansênicas e recidivas da doença. Dessa forma, torna-se preciso refletir nas ações programáticas de saúde da mulher, tendo como foco o planejamento sexual e reprodutivo, atenção humanizada na gestação, parto e puerpério.

Para Araújo \& Lana (2020), a pobreza e as desigualdades sociais devem ser consideradas no processo endêmico da hanseníase, bem como é preciso ampliar programas sociais, acreditando-se que a melhoria das condições de vida da população é fundamental para interromper a cadeia de transmissão da doença.

A Tabela 2 apresenta o perfil clínico-epidemiológico das participantes, dos quais $38,10 \%(\mathrm{n}=8)$ foram classificadas na forma clínica indeterminada, destaca-se o alto percentual de casos não classificados que foi de $19,05 \%$ ( $\mathrm{n}=4$ ). No que concerne a classificação operacional, em sua maioria foram casos paucibacilares com $66,67 \%(n=14)$.

Tabela 2: Perfil clínico- epidemiológico das mulheres pós-alta de hanseníase participantes do estudo. Rio Largo-AL, Brasil, 2019 ( $\mathrm{n}=21)$.

\begin{tabular}{llll}
\hline Variáveis & Categorias & $\mathbf{N}$ & \% \\
\hline \multirow{3}{*}{ Forma clínica } & Indeterminada & 8 & 38,10 \\
& Dimorfa & 4 & 19,05 \\
& Tuberculoide & 5 & 23,81 \\
& Não classificado & 4 & 19,05 \\
\hline \multirow{2}{*}{ Classificação operacional } & Paucibacilar & 14 & 66,67 \\
& Multibacilar & 7 & 33,33 \\
\hline
\end{tabular}

Fonte: SINAN (2019).

De acordo com dados do Ministério da Saúde, na cidade de Rio Largo, foram notificados 55 casos de hanseníase de 2016 a 2020, sendo que 67,3\% (n=37) foram diagnosticados na forma multibacilar (DATASUS, 2021). Como este estudo tratou-se de uma amostra em mulheres pós-alta, em sua maioria foram as paucibacilares. Além disso, dos casos de hanseníase de 2016 a 2020 de Rio Largo, cerca de 23,6\% (n=13) foram na forma clínica dimorfa (DATASUS, 2021), resultados também diferentes deste estudo.

Em relação ao grau de incapacidade física, destacou-se que 66,67\% (n=14) apresentaram grau 0 de incapacidade física no diagnóstico, e 47,62\% (n=10) das mulheres não foram avaliadas na alta (Tabela 3). 
Tabela 3: Avaliação do grau de incapacidade física das mulheres pós-alta de hanseníase participantes do estudo. Rio Largo-AL, Brasil, 2019 ( $\mathrm{n}=21)$.

\begin{tabular}{llll}
\hline Variáveis & Categorias & n & \% \\
\hline \multirow{2}{*}{$\begin{array}{l}\text { Grau de incapacidade física no momento do } \\
\text { diagnóstico }\end{array}$} & Grau 0 & 14 & 66,67 \\
& Grau I & 4 & 19,05 \\
& Grau II & 1 & 4,76 \\
& Não avaliado & 2 & 9,52 \\
\hline \multirow{2}{*}{ Grau de incapacidade física no momento da alta } & Grau 0 & 9 & 42,86 \\
& Grau I & 2 & 9,52 \\
& Não avaliado & 10 & 47,62 \\
\hline
\end{tabular}

Fonte: SINAN (2019).

A avaliação do grau de incapacidade, tanto no diagnóstico como na alta, avaliam a qualidade do atendimento nos serviços de saúde (Brasil, 2016a; Santana et al., 2018). Estudo realizado na Paraíba, destacou a importância do acompanhamento periódico para prevenir o surgimento/agravamento de deformidades, sendo importante criar protocolos de seguimento dos indivíduos após a alta da poliquimioterapia (Santana et al., 2018). Além de avaliar a qualidade de atendimento, a avaliação do grau de incapacidade é indicador de qualidade de vida e participação social, pois o diagnostico com sequelas indica detecção tardia. A não avaliação do grau de incapacidade também pode sinalizar fragilidade do serviço em acompanhar os pacientes no pós- alta por cura, dessa maneira é preciso fortalecer essas ações, principalmente nos serviços de atenção primária por serem porta de entrada preferencial dos usuários.

No que concerne as mulheres, a presença da incapacidade física apresenta-se como uma dupla carga, pois o ser feminino é carregado de padronização da beleza. Dessa maneira, é preciso refletir em estratégias que desmistifiquem os padrões de beleza impostos pela sociedade, e independente das características físicas, toda mulher merece ser respeitada e admirada pelas suas singularidades.

A Tabela 4 apresenta a avaliação neurofuncional de nariz e olhos em mulheres na pós- alta de hanseníase. É possível observar que na avaliação do nariz houveram poucas queixas, apenas 9,52\% (n=2) queixaram-se de ressecamento. Na avaliação do olho, constatou-se $23,81 \%(n=5)$ a presença de catarata e $71,43 \%(n=15)$ acuidade visual preservada. 
Tabela 4: Avaliação neurofuncional de nariz e olhos em mulheres pós-alta de Hanseníase participantes do estudo. Rio Largo-AL, Brasil, 2019 ( $\mathrm{n}=21)$.

\begin{tabular}{|c|c|c|c|c|}
\hline Componente & Variável & Categorias & $\mathbf{n}$ & $\%$ \\
\hline \multirow{4}{*}{ Nariz } & \multirow{2}{*}{ Ressecamento do nariz } & Com queixa & 2 & 9,52 \\
\hline & & Sem queixa & 19 & 90,48 \\
\hline & Ferida no nariz & Ausência & 21 & 100,00 \\
\hline & Perfuração de septo no nariz & Ausência & 21 & 100,00 \\
\hline \multirow{8}{*}{ Olhos } & Triquíase & Ausência & 21 & 100,00 \\
\hline & Ectrópio & Ausência & 21 & 100,00 \\
\hline & \multirow{2}{*}{ Catarata } & Ambos os lados & 5 & 23,81 \\
\hline & & Não apresenta & 16 & 76,19 \\
\hline & \multirow[t]{2}{*}{ Acuidade visual preservada } & Ambos os lados & 15 & 71,43 \\
\hline & & Não apresenta & 6 & 28,57 \\
\hline & \multirow[t]{2}{*}{ Anestesia da córnea } & Ambos os lados & 6 & 28,57 \\
\hline & & Não apresenta & 15 & 71,43 \\
\hline
\end{tabular}

Fonte: Autores (2019).

Para Santana et al. (2018), apesar de não serem quantificadas para determinação da incapacidade física, as lesões no nariz apresentam importância tanto no aspecto físico devido as deformidades, quanto no psicológico pelo estigma associado à doença, posto que este é considerado determinante nas relações interpessoais. Assim, as vidas das mulheres acometidas pela doença são impactadas, principalmente no trabalho e na realização das atividades diárias (Gonçalves, et al., 2018).

$\mathrm{Na}$ avaliação neurofuncional dos olhos, as mulheres avaliadas apresentaram poucas alterações oftalmológicas. No entanto, torna-se fundamental a avaliação periódica da força muscular e de sensibilidade dos olhos, pois é importante fonte de informação acerca do nível de acometimento neural (Santana et al., 2017).

$\mathrm{Na}$ avaliação dos membros superiores, observou-se que a maioria das mulheres não apresentaram acometimento nos nervos ulnar, mediano e radial, conforme dados mostrados na tabela 5. A menor evidência de lesões nas mãos pode ser justificada pelo maior autocuidado e percepção precoce dos problemas incapacitantes nas mãos, o que não ocorre com os pés (Santana et al., 2017).

$\mathrm{Na}$ avaliação dos membros superiores, observou-se que a maioria das mulheres não apresentaram acometimento nos nervos ulnar, mediano e radial, conforme dados mostrados na tabela 5. A menor evidência de lesões nas mãos pode ser justificada pelo maior autocuidado e percepção precoce dos problemas incapacitantes nas mãos, o que não ocorre com os pés (Santana et al, 2017). 
Tabela 5: Avaliação neurofuncional de membros superiores em mulheres pós-alta de Hanseníase participantes do estudo. Rio Largo-AL, Brasil, 2019 ( $\mathrm{n}=21$ ).

\begin{tabular}{|c|c|c|c|}
\hline Variável & Categorias & $\mathbf{N}$ & $\%$ \\
\hline \multirow{2}{*}{ Nervo ulnar direito } & Normal & 13 & 61,90 \\
\hline & Dor & 8 & 38,10 \\
\hline \multirow{5}{*}{ Nervo ulnar esquerdo } & Normal & 11 & 52,38 \\
\hline & Espessado & 1 & 4,76 \\
\hline & Dor & 6 & 28,57 \\
\hline & Formigamento & 2 & 9,52 \\
\hline & Anestesia & 1 & 4,76 \\
\hline \multirow{3}{*}{ Nervo radial esquerdo } & Normal & 14 & 66,67 \\
\hline & Espessado & 2 & 9,52 \\
\hline & Dor & 5 & 23,81 \\
\hline \multirow{2}{*}{ Abdução do quinto dedo direito } & Forte & 14 & 66,67 \\
\hline & Diminuído & 7 & 33,33 \\
\hline \multirow{3}{*}{ Abdução do quinto dedo esquerdo } & Forte & 11 & 52,38 \\
\hline & Diminuído & 9 & 42,86 \\
\hline & Paralisado & 1 & 4,76 \\
\hline Abdução do polegar & Forte & 21 & 100,00 \\
\hline Extensão de punho direito & Forte & 21 & 100,00 \\
\hline \multirow{2}{*}{ Extensão de punho esquerdo } & Forte & 16 & 76,19 \\
\hline & Diminuído & 5 & 23,81 \\
\hline \multirow{3}{*}{ Diminuição de sensibilidade dos MMSS } & Esquerdo & 1 & 4,76 \\
\hline & Ambos os lados & 2 & 9,52 \\
\hline & Não apresenta & 18 & 85,71 \\
\hline
\end{tabular}

Fonte: Autores (2019).

$\mathrm{Na}$ avaliação dos membros inferiores, no geral, não foram observados acometimentos nos nervos tibial e fibular das mulheres no pós- alta por cura, conforme dados da tabela 6. No entanto, destacou-se a sensação de "dor" autorreferida pelas mulheres avaliadas. Estudo realizado por Gonçalves et al. (2018) enfatizou que a dor física interfere na realização do trabalho e nas atividades diárias das mulheres. Além disso, dor associada a problemas na autoimagem, afetam diretamente diversos aspectos da vida das mulheres.

Assim as ações de autocuidado devem ser encorajadas pelos profissionais de saúde, pois é uma estratégia fundamental no tratamento da doença e na prevenção de incapacidades para evitar agravamento (Brasil, 2008). As lesões neurais juntamente com a perda de sensibilidade protetora, pode ter como consequência queimaduras, ferimentos, úlceras, fissuras, atrofias, articulações rígidas e desequilíbrio muscular, tornando-se imprescindível avaliação periódica e realização de autocuidado. 
Tabela 6: Avaliação neurofuncional de membros inferiores em mulheres pós-alta de Hanseníase participantes do estudo. Rio Largo-AL, Brasil, 2019 ( $\mathrm{n}=21$ ).

\begin{tabular}{llll}
\hline Variável & Categoria & $\mathbf{n}$ & \% \\
\hline \multirow{2}{*}{ Nervo fibular direito } & Normal & 14 & 66,67 \\
& Espessado & 1 & 4,76 \\
& Dor & 5 & 23,81 \\
\hline \multirow{2}{*}{ Nervo fibular esquerdo } & Normal & 12 & 57,14 \\
& Espessado & 1 & 4,76 \\
& Dor & 7 & 33,33 \\
Nervo tibial direito & Normal & 11 & 52,38 \\
& Espessado & 1 & 4,76 \\
& Dor & 7 & 33,33 \\
& Formigamento & 1 & 4,76 \\
\hline \multirow{2}{*}{ Nervo tibial esquerdo } & Normal & 10 & 47,62 \\
& Dor & 7 & 33,33 \\
\hline
\end{tabular}

Fonte: Autores (2019).

Evidencia-se a dificuldade dos serviços de acompanhar os pacientes no pós-alta de hanseníase, pois uma adequada assistência previne as incapacidades físicas e melhora a qualidade de vida (Monteiro et al, 2013). Além disso, torna-se preciso formar grupos de autocuidado e cuidados holísticos no pós-alta das pessoas atingidas pela hanseníase, ao agregar novo enfoque para os profissionais de saúde e os serviços de saúde em relação a essa patologia (Lima et al., 2021).

Nesse sentido, algumas atividades de prevenção no pós-alta de hanseníase devem ser encorajadas, tais como hidratação da pele, lubrificação, cuidado com unhas e calosidades, inspeção de calçados antes de usá-los, uso de palmilhas ou sapatos adaptados e evitar transitar em ambientes escuros e com desníveis de pisos. Ademais é preciso transcender a prática assistencial centrada na doença e incorporar no programa a prevenção de incapacidades físicas, com ampliação das ações para o âmbito social, familiar e cultural (Souza et al., 2020).

No tocante das mulheres, ressalta-se a necessidade de atenção com aquelas em idade reprodutiva pelo risco de recidivas, exacerbação de lesões pré-existentes e progressão nas formas clínicas, principalmente em gestantes ou lactantes. Essas condições exigem acompanhamento especial das mulheres portadoras de hanseníase, no sentido de prepará-las para prática anticonceptiva segura e a prevenção de gestação de alto risco (Tavares, 2014).

\section{Conclusão}

Constatou-se fragilidade do serviço em acompanhar as mulheres no pós- alta por cura de hanseníase, além disso, na avaliação neurofuncional, foi encontrada maior ocorrência de alterações sensitivas em comparação as motoras e também ausência de deformidades físicas. Destaca-se a importância de fortalecer os cuidados mesmo após a alta da poliquimioterapia, pois é preciso prevenir as incapacidades e realizar o manejo das incapacidades residuais. Sendo importante sistematizar o acompanhamento dos pacientes no pós- alta por cura, com assistência periódica e fortalecimento da vigilância em saúde.

Torna-se necessário realizar estudos que identifique as dificuldades para o monitoramento e assistência das mulheres que foram acometidas pela hanseníase, tendo em vista as repercussões psicológicas, físicas e sociais que a doença pode causar. Obteve-se como limitação do estudo dificuldade em localizar as mulheres no pós-alta de hanseníase, e assim, como 
consequência uma baixa representativa na amostra. No entanto, os resultados podem direcionar medidas específicas e apropriadas para o público feminino acometido pela hanseníase.

Dessa forma, percebe-se o quanto a hanseníase ainda carece de amplos estudos, sobretudo em relação às pessoas mais vulneráveis, como é o caso das mulheres, mas há outros grupos que sugerimos pesquisas como essas, como: em menores de quinze anos, presidiários, asilados e demais grupos que convivem em aglomerados habitacionais. Tais pesquisas promovem repercussões positivas não só para os portadores de hanseníase, mas também para a Saúde Pública brasileira na luta pela erradicação da hanseníase.

\section{Agradecimentos}

Agradecemos à Secretaria municipal de Rio Largo-AL, às enfermeiras das Unidades Básicas de Saúde visitadas e ao programa institucional de bolsas de iniciação científica 2018-2019 da Universidade Federal de Alagoas (UFAL) pela efetivação da pesquisa.

\section{Referências}

Alagoas. (2015). Saúde Alagoas: Análise da Situação de Saúde. Secretaria de Estado da Saúde de Alagoas, 2014. http://www.saude.al.gov.br/wpcontent/uploads/2016/04/documento_tecnico_08-01-2015_16-19-33_1\%C2\%BA_Regiao_ASS_2014.pdf.

Alagoas. (2019). Ação de saúde orienta população sobre hanseníase na orla de Maceió. 2019. http://www.saude.al.gov.br/2019/01/21/acao-de-saude-orientapopulacao-sobre-hanseniase-na-orla-da-ponta-verde/.

Araújo, K. M. F. A. \& Lana, F. C. F. (2020). Relação da hanseníase com a cobertura da estratégia saúde da família e condições socioeconómicas. Ciencia y Enfermeria, 26(1), 1-9. https://dx.doi.org/10.4067/s0717-95532020000100201.

Ávila e Silva, L. M., \& Barsaglini, R. A. (2018). “A reação é o mais difícil, é pior que hanseníase”: contradições e ambiguidades na experiência de mulheres com reações hansênicas. Physis: Revista de Saúde Coletiva. 28(4),1-19. https://doi.org/10.1590/S0103-73312018280422.

Bonita, R., Beaglehole R., \& Kjellstrom T. (2010). Epidemiologia Básica. São Paulo: Santos.

Capp, E., \& Nienov, O. H. (Orgs). (2020). Bioestatística quantitativa e aplicada. Universidade Federal do Rio Grande do Sul. Faculdade de Medicina. Programa de Pós-Graduação em Ciências da Saúde: Ginecologia e Obstetrícia. Porto Alegre: UFRGS. https://lume.ufrgs.br/bitstream/handle/10183/213116/001117616.pdf?sequence=1\&isAllowed=y.

DATASUS (2021). Ministério da Saúde. Epidemiológicas e morbidade. https://datasus.saude.gov.br/acesso-a-informacao/casos-de-hanseniase-desde-2001sinan/.

Freire, M. C. M., \& Pattussi M. P. Tipos de estudos. IN: ESTRELA, C. Metodologia científica. Ciência, ensino e pesquisa. $3^{\text {a }}$ ed. Porto Alegre: Artes Médicas, 2018. p.109-127.

Gonçalves, M., Prado, M. A. R., Silva, S. S., Santos, K. S., Araujo, P. N. \& Fortuna, C. M. (2018). Work and Leprosy: women in their pains, struggles and toils. Revista Brasileira de Enfermagem. 71(Suppl 1):660-667. https://doi.org/10.1590/0034-7167-2017-0598.

Gonçalves, M., Santos, K. S., Silva, S. S., Marcussi, T. C. C., Carvalho, K. V., \& Fortuna, C. M. (2021). Women and leprosy: interferences and experiences. Revista Latino-Americana de Enfermagem. 29: e3419. https://doi.org/10.1590/1518-8345.4347.3419.

Instituto Brasileiro de Geografia e Estatística. IBGE (2021). https://cidades.ibge.gov.br/brasil/al/rio-largo/panorama.

Lages, D. S., Kerr, B. M., Bueno, I. C., Niitsuma, E. N., \& Lana, F. C. (2018). A baixa escolaridade está associada ao aumento de incapacidades físicas no diagnóstico de hanseníase no Vale do Jequitinhonha. HU Revista. 44(3): 303-309. https://doi.org/10.34019/1982-8047.2018.v44.14035.

Leal, D. R., Cazarin, G., Bezerra, L. C. A., Albuquerque, A. C., \& Felisberto, E. (2017). Programa de Controle da Hanseníase: uma avaliação da implantação no nível distrital. Saúde debate. 41(spe): 209-228. https://doi.org/10.1590/0103-11042017S16.

Lima, A. B. A., Tavares, C. M., Santos, I. M. R., Bragagnollo, G. R., Gomes, N. M. C., \& Santos, A. A. P. (2021). Mulheres no pós-alta de hanseníase: aspectos clínicos, sociodemográficos e reprodutivos. Research, Society and Development. 10 (1): e13210111369. https://doi.org/10.33448/rsd-v10i1.11369.

Ministério da Saúde. (2008). Manual de prevenção de incapacidades: Cadernos de prevenção e reabilitação em hanseníase. Departamento de Vigilância Epidemiológica. https://bvsms.saude.gov.br/bvs/publicacoes/manual_prevencao_incapacidades.pdf.

Ministério da Saúde. (2016). Diretrizes para vigilância, atenção e eliminação da Hanseníase como problema de saúde pública: manual técnico-operacional Secretaria de Vigilância em Saúde. Departamento de Vigilância das Doenças Transmissíveis. https://portalarquivos2.saude.gov.br/images/pdf/2016/fevereiro/04/diretrizes-eliminacao-hanseniase-4fev16-web.pdf. 
Ministério da Saúde. (2021). Boletim Epidemiológico da Hanseníase. Secretaria de Vigilância em Saúde. http://www.aids.gov.br/pt-br/pub/2021/boletimepidemiologico-hanseniase-2021.

Monteiro, L. D., Alencar, C. H. M., Barbosa, J. C., Braga, K. P., Castro, M. D., \& Heukelbach, J. (2013). Incapacidades físicas em pessoas acometidas pela hanseníase no período pós-alta da poliquimioterapia em um município no Norte do Brasil. Caderno de Saúde Pública. 29(5): 909-920. https://doi.org/10.1590/S0102-311X2013000500009.

Monteiro, L. D., Mota, R. M. S., Martins-Melo, F. R., Alencar, C. H., \& Heukelbach, J. (2017). Determinantes sociais da hanseníase em um estado hiperendêmico da região Norte do Brasil. Revista de Saúde Pública. 51(70): 1-11. https://doi.org/10.1590/S1518-8787.2017051006655.

Pinheiro, M. G. C., Lins, S. L. F., Gomes, B. R. S., Simpson, C. A., Mendes, F. R. P., \& Miranda, F. A. N. (2019). Contextual analysis of health care at discharge in leprosy: an integrative review. Revista Gaúcha de Enfermagem. 40:e20180258. https://doi.org/10.1590/1983-1447.2019.20180258.

Rodrigues, R. N., Niitsuma, E. N. A., Bueno, I. C., Baquero, O. S., Jardim, C. C. G., \& Lana, F. C. F. (2017). Leprosy and health vulnerability in Belo Horizonte, Minas Gerais. Revista Mineira de Enfermagem. 21:e-997. http://www.dx.doi.org/10.5935/1415-2762.20170007.

Santana, E. M. F., Antas, E. M. V., Brito, K. K. G., \& Silva, M. A. (2017). Profile of leprosy patients in a secondary health care center. J Nurs UFPE on line. 11(11):4404-4409. https://periodicos.ufpe.br/revistas/revistaenfermagem/article/view/14278/24707.

Santana, E. M. F., Brito, K. K. G., Nogueira, J. A., Leabedal, O. D. C. P., Costa, M. M. L., Silva, M. A., \& Soares, M. J. G. O. (2018). Deficiências e incapacidades na hanseníase: do diagnóstico à alta por cura. Revista Eletrônica de Enfermagem. 20 (v20a15): 1-11. https://www.revistas.ufg.br/fen/article/view/50436.

Souza, C. D. F., Medronho, R. A., Santos, F. G. B., Magalhães, M. A. F. M., \& Luna, C. F. (2020). Modelagem espacial da hanseníase no estado da Bahia, Brasil (2001 a 2015) e determinantes sociais da saúde. Ciência e saúde coletiva. 25 (8): 2915-2926. https://doi.org/10.1590/1413-81232020258.21522018.

Tavares, C. M. (2014). A saúde reprodutiva de mulheres portadoras e ex-portadoras de hanseníase em uma capital do Nordeste. 124p. Tese (Doutorado), Escola de Enfermagem de Ribeirão Preto/USP.

World Health Organization. (2021). Weekly epidemiological record. Global leprosy (Hansen disease) update, 2020: impact of COVID-19 on global leprosy control. http://www.who.int/wer. 